\title{
Vascular Endothelial Growth Factor-A Levels in Term Neonates
}

\author{
Yogavijayan Kandasamy ${ }^{1,2,3}$ Leo Hartley ${ }^{4}$ Donna Rudd ${ }^{3}$
}

${ }^{1}$ Department of Neonatology, Townsville Hospital, Queensland, Australia

2 Mothers and Babies Research Centre, Hunter Medical Research Institute, John Hunter Hospital, The University of Newcastle, Newcastle, New South Wales, Australia

${ }^{3}$ College of Public Health, Medical and Veterinary Sciences, James Cook University, Douglas, Queensland, Australia

${ }^{4}$ Department of Optometry and Vision Science, The University of Melbourne, Victoria, Australia

J Child Sci 2017;7:e151-e154.

\begin{abstract}
Address for correspondence Yogavijayan Kandasamy, MBBS, FRACP, PhD, Department of Neonatology, Townsville Hospital, 100 Angus Smith Drive, Douglas, Queensland 4814, Australia (e-mail: Yoga.Kandasamy@health.qld.gov.au).
\end{abstract}

\author{
Abstract \\ Keywords \\ - vascular endothelial \\ growth factor \\ - term \\ - neonates
}

Vascular endothelial growth factor-A (VEGF-A) plays an integral role in physiological and pathophysiological angiogenesis and has increasingly been implicated in the development of retinopathy of prematurity (ROP) in preterm infants. Application of intravitreal anti-VEGF is frequently used to treat ROP with little consideration given to the role of VEGF-A in neonatal growth and development. Previous studies have demonstrated systemic anti-VEGF persistence, reduced peripheral VEGF levels following treatment, and possible diagnostic and prognostic uses for VEGF-A determination. This study seeks to determine a normal range for serum VEGF-A (sVEGF-A) in healthy, term infants. The sVEGF-A levels were obtained from 32 neonates born at term infants (16 males and 16 females) using an enzyme-linked immunosorbent assay. No significant correlations were found between SVEGF-A levels and time of sample collection, birth weight, or gender. The median sVEGF-A level was 976 (394-1635) $\mathrm{pg} / \mathrm{mL}$ (95\% confidence interval for median: $496-1,318 \mathrm{pg} / \mathrm{mL}$ ). This preliminary study determines a normal range for the sVEGF-A level in healthy, term neonates. This normal range will provide a tool to assist in the diagnosis, prognosis, and monitoring of treatment of infants with ROP.

\section{Introduction}

Vascular endothelial growth factor (VEGF), a versatile proangiogenic growth factor was first described by Leung et al, ${ }^{1}$ although Senger et al first identified this protein 6 years earlier but called it vascular permeability factor. ${ }^{2-4}$ The VEGF family in humans consists of VEGF-A, placental growth factor(PLGF), VEGF-B, VEGF-C, and VEGF-D. All are induced during hypoxic stress in mammalian cells. ${ }^{5}$ VEGF-A is a homodimeric protein and is believed to be a critical mediator of vasculogenesis during embryogenesis. In humans, the gene encoding VEGF-A is located on chromosome number 6 (6p12). ${ }^{5}$ VEGF is thought to act on endothelial cells to bring about numerous effects, including induction of angiogenesis, increased vascular permeability, and endothelial cell growth, through the promotion of cell migration and inhibiting apoptosis. VEGF and notch signaling engage in an intricate crosstalk to balance tip and stalkcell formation and regulate directed tip cell migration and stalk cell proliferation. ${ }^{6}$

Vascular endothelial growth factor plays a central role in both physiological and pathological angiogenesis and neovascularization. ${ }^{7,8}$ The soluble forms of VEGF-A are the main received

July 11,2017

accepted after revision

October 30, 2017
DOI https://doi.org/

10.1055/s-0037-1612596. ISSN 2474-5871.
Copyright (c) 2017 Georg Thieme Verlag License terms

KG Stuttgart · New York 
isoforms that induce paracrine activation and proliferation of endothelial cells through binding to the specific VEGF tyrosine kinase receptors, VEGFR1 and VEGFR2 (predominantly). ${ }^{7}$ VEGF binding produces receptor activation and an upregulation of receptor-related kinase activity, allowing for phosphorylation of tyrosine residues and further upregulation of cell signaling pathways, the mechanisms behind which remain to be fully elucidated. ${ }^{7}$

Three VEGF tyrosine kinase receptors have been identified; FMS-like tyrosine kinase Flt-1 (VEGFR1/flt-1), VEGFR2/ KDR/Flk-1, and Flt-4/VEGFR3. VEGFR-1 has a high affinity for VEGF-A and appears to modulate endothelial cell division at the earliest stages of vascular development just before the development of blood vessels. Through a complex series of events, hypoxia upregulates both VEGF-A and VEGFR1 production via hypoxia-inducible factor-1, a protein complex which binds to enhancer sequences of the VEGF-A gene. Various studies have also described the upregulation of VEGFR2 following hypoxia. ${ }^{7}$

Retinopathy of prematurity (ROP), a retinal vasculoproliferative disease that is exclusive to preterm neonates, has become an important cause of preventable childhood blindness worldwide. ${ }^{9,10}$ The established and traditional way to treat this condition is by using laser photocoagulation to ablate vast areas of peripheral retina thereby reducing hypoxic drive and thus the retinopathy. However, over the past 7 years, intravitreal VEGF inhibitor (anti-VEGF) therapy has become an alternative treatment option for some clinicians. ${ }^{11}$ Intravitreally injected anti-VEGF has been subsequently detected in the systemic circulation in neonates. ${ }^{12,13}$

The level of serum VEGF-A (sVEGF-A) in term neonates has not been well established. There has been a difference noted between serum and plasma VEGF-A levels. ${ }^{14}$ Despite the lack of this knowledge, clinicians are increasingly using anti-VEGF to treat ROP. Hence, we performed a preliminary study to determine the normal sVEGF-A level in term neonates.

\section{Materials and Methods}

This prospective study was conducted in the Department of Neonatology, Townsville Hospital, Queensland, Australia. The Department of Neonatology is a tertiary perinatal center that caters for a region with more than 10,000 births each year. The study commenced in July 2014, and the data presented in this report are based on patients who were recruited until July 2016. The patients recruited in this study are part of a larger ongoing study that investigates the relationship among prematurity, retinal vascularization, and renal development in preterm and low birth weight neonates. The term, healthy neonates (gestation $>37$ weeks) who presented to the hospital with minor neonatal conditions, such as neonatal jaundice, feeding problems, and risk of infection, were eligible to be part of this study. Neonates with congenital abnormalities or syndromes and those needing respiratory support or surgery were excluded. Consent was obtained from the parents of all participants included in the study. This study was approved by the Townsville Health District Human Research Ethics Committee.

\section{VEGF Measurement}

Peripheral venous samples were obtained from neonates born at term (completed 37 weeks' gestation) for VEGF measurement. Samples were transported and processed promptly (within 60 minutes).VEGF measurement was performed using a VEGF human enzyme-linked immunosorbent assay (ELISA) kit (ab100662, Abcam) which detects both VEGF-A165 and VEGF-A121 isoforms with a sensitivity of $10 \mathrm{pg} / \mathrm{mL}$ and an intra-assay precision (coefficient of variation [CV] \%) of $<10 \%$ and interassay precision (CV\%) of $<12 \%$.

Statistical analyses were performed using MedCalc Version 15.6 (MedCalc Software bvba, Mariakerke, Belgium). The normality of the variables was determined using the D'AgostinoPearson's test. ${ }^{15}$ Data are presented as mean (standard deviation [SD]) or as median (interquartile range[IQR]). Student's $t$-test or Mann-Whitney's test was used where appropriate, with $p$-values $<0.05$ considered significant.

\section{Findings}

Thirty-two term neonates were recruited, with a mean gestational age of 38.8 (1.2) weeks, and a mean birth weight of 3,161 (648) $\mathrm{g}$. The mean postnatal age for these infants at the time of sampling was 6.9 (4.3) days. The median sVEGF-A level was 976 (394-1,635) pg/mL (95\% confidence interval [CI] for median: $496-1,318 \mathrm{pg} / \mathrm{mL}$ ). Sixteen of the infants were females, and there was no significant difference in SVEGF-A level between female and male infants (1,318 [498-2,046] vs. 793 [294-1,206] $\mathrm{pg} / \mathrm{mL} ; p=0.073)$. There was no correlation between birth weight and plasma VEGF-A levels (-Fig. 1). There was also no significant correlation between time of sampling and VEGF-A level $(r=0.04, p=0.0832)$.

\section{Discussion}

There have been relatively few studies that have reported sVEGF-A levels in infants and less reporting of sVEGF-A levels in healthy term neonates. To our knowledge, this is the first study to define the 95\% CI for VEGF-A serum levels in healthy,

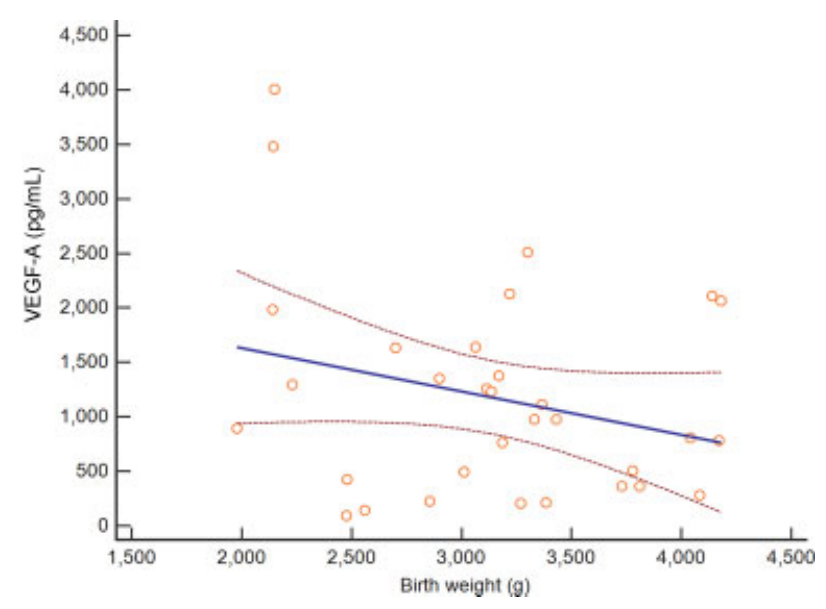

Fig. 1 No correlation between birth weight and VEGF-A level $(y=2,421.6681+x=-0.3959 ; p=0.135)$. VEGF-A, vascular endothelial growth factor $A$. 
term neonates. Lassus et a ${ }^{16}$ have previously published plasma VEGF levels in a cohort of 13 term infants $(138.5 \pm 39.4 \mathrm{pg} / \mathrm{mL})$.

Previously published human studies in premature neonates have produced contradictory findings. A few have also demonstrated increased concentrations of serum VEGF in preterm neonates associated with certain conditions such as ROP when compared with their normal counterparts. ${ }^{17-20}$ Yenice et al found serum VEGF levels taken from cord blood at birth to be lower in neonates who later developed ROP. ${ }^{21}$ VEGF antagonists are increasingly used as a clinical treatment in neonates. The measurement of VEGFA levels in the blood has been extensively investigated as a potential diagnostic biomarker. We believe the knowledge of normal levels in healthy, term infants is essential to aid clinical interpretation, prognostic, and diagnostic decision making.

There are some limitations to our study. It is important to note that VEGF-A measurement and interpretation can be significantly affected by some preanalytical variables, such as the sample type, serum versus plasma, and the method of analysis multiplexed immunoassay versus ELISA. ${ }^{22}$ In this study, serum samples were analyzed using a standard ELISA assay. The $95 \% \mathrm{Cl}$ for median 496 to $1,318 \mathrm{pg} / \mathrm{mL}$ is comparable to that reported by other studies. ${ }^{12,13,21,23-25}$ This $\mathrm{CI}$ for serum VEGF-A is higher than that reported by Lassus et al from their analysis of plasma VEGF. ${ }^{16}$ Serum samples may have a higher concentration of VEGF-A possibly due to the release of plateletderived VEGF-A. ${ }^{14}$ It is also unclear if systemic VEGF-A measurement accurately reflects the biological effect of VEGF-A, as VEGF signaling is increasingly recognized for its function outside of the vascular system, notably during neural development, and blood vessels regulate epithelial branching morphogenesis. ${ }^{26}$ Another limitation of our study is the variability in the infants' postnatal age. However, this study did not find any significant correlation between age at the time of sampling and VEGF-A level. Some of the infants in our cohort required phototherapy for jaundice, and there are data to suggest that phototherapy may influence serum VEGF levels. ${ }^{27}$

\section{Conclusion}

This study reports a $95 \% \mathrm{CI}$ for serum VEGF-A levels on healthy term infants. The sVEGF-A levels were independent of age at the time of sample collection, birth weight, and gender. This normal reference range may serve as a tool for assisting the diagnosis, prognosis, and effects of anti-VEGF treatment. However, it has to be appreciated that there may be a difference between serum and plasma levels of VEGF-A and that VEGF-A is being recognized for its function outside of the vascular system.

Note

Consent was obtained from parents of all participants included in the study. This study was approved by the Townsville Health District Human Research Ethics Committee.

\section{Authors' Contributions}

Y.K. was involved in the study design, sample collection, data analysis, and preparation of manuscript and approval of final draft. D.R. was involved in sample analysis, data interpretation, manuscript preparation, and approval of final draft. L.H. was involved in study design, data analysis, manuscript preparation, and approval of final draft.

\section{Funding}

This study was funded by the National Health and Medical Research Council, Australia.

Conflict of Interest

None.

\section{References}

1 Leung DW, Cachianes G, Kuang WJ, Goeddel DV, Ferrara N. Vascular endothelial growth factor is a secreted angiogenic mitogen. Science 1989;246(4935):1306-1309

2 Ferrara N. VEGF and the quest for tumour angiogenesis factors. Nat Rev Cancer 2002;2(10):795-803

3 Caldwell RB, Bartoli M, Behzadian MA, et al. Vascular endothelial growth factor and diabetic retinopathy: pathophysiological mechanisms and treatment perspectives. Diabetes Metab Res Rev 2003;19(06):442-455

4 Ferrara N. VEGF-A: a critical regulator of blood vessel growth. Eur Cytokine Netw 2009;20(04):158-163

5 Ramakrishnan S, Anand V, Roy S. Vascular endothelial growth factor signaling in hypoxia and inflammation. J Neuroimmune Pharmacol 2014;9(02):142-160

6 Blanco R, Gerhardt H. VEGF and notch in tip and stalk cell selection. Cold Spring Harb Perspect Med 2013;3(01):a006569

7 Ferrara N, Gerber HP, LeCouter J. The biology of VEGF and its receptors. Nat Med 2003;9(06):669-676

8 McColm JR, Geisen P, Hartnett ME. VEGF isoforms and their expression after a single episode of hypoxia or repeated fluctuations between hyperoxia and hypoxia: relevance to clinical ROP. Mol Vis 2004;10:512-520

9 Hartnett ME. Pathophysiology and mechanisms of severe retinopathy of prematurity. Ophthalmology 2015;122(01):200-210

10 Blencowe H, Lawn JE, Vazquez T, Fielder A, Gilbert C. Pretermassociated visual impairment and estimates of retinopathy of prematurity at regional and global levels for 2010. Pediatr Res 2013;74(Suppl 1):35-49

11 Hartnett ME. Vascular endothelial growth factor antagonist therapy for retinopathy of prematurity. Clin Perinatol 2014;41(04): 925-943

12 Sato T, Wada K, Arahori H, et al. Serum concentrations of bevacizumab (Avastin) and vascular endothelial growth factor in infants with retinopathy of prematurity. Am J Ophthalmol 2012;153(02): 327-333.e1

13 Wu WC, Lien R, Liao PJ, et al. Serum levels of vascular endothelial growth factor and related factors after intravitreous bevacizumab injection for retinopathy of prematurity. JAMA Ophthalmol 2015; 133(04):391-397

14 Mcllhenny C, George WD, Doughty JC. A comparison of serum and plasma levels of vascular endothelial growth factor during the menstrual cycle in healthy female volunteers. Br J Cancer 2002;86 (11):1786-1789

15 D'Agostino RB, Belanger A, D'Agostino RB Jr. A suggestion for using powerful and informative tests of normality. Am Stat 1990;44 (04):316-321

16 Lassus P, Turanlahti M, Heikkilä P, et al. Pulmonary vascular endothelial growth factor and Flt-1 in fetuses, in acute and chronic lung disease, and in persistent pulmonary hypertension of the newborn. Am J Respir Crit Care Med 2001;164(10 Pt 1):1981-1987

17 Machalińska A, Modrzejewska M, Dziedziejko V, et al. Evaluation of VEGF and IGF-1 plasma levels in preterm infants-potential 
correlation with retinopathy of prematurity, clinical implications [in Polish]. Klin Oczna 2009;111(10-12):302-306

18 Hellgren G, Löfqvist C, Hård AL, et al. Serum concentrations of vascular endothelial growth factor in relation to retinopathy of prematurity. Pediatr Res 2016;79(1-1):70-75

19 Du J, Chen C, Shi WJ. Changes of serum vascular endothelial growth factor in newborn infants and its relationship with retinopathy of prematurity [in Chinese]. Zhonghua Yi Xue Za Zhi 2010;90(28):1982-1985

20 Goswami B, Goyal M, Beri S, Garg R, Saili A, Jain A. Role of serum levels of vascular endothelial growth factor and its receptor in retinopathy of prematurity. Iran J Pediatr 2015;25(04):e2373

21 Yenice O, Cerman E, Ashour A, et al. Serum erythropoietin, insulin-like growth factor 1, and vascular endothelial growth factor in etiopathogenesis of retinopathy of prematurity. Ophthalmic Surg Lasers Imaging Retina 2013;44(06):549-554

22 Walz JM, Boehringer D, Deissler HL, et al. Pre-analytical parameters affecting vascular endothelial growth factor measurement in plasma: identifying confounders. PLoS One 2016;11(01): e0145375

23 Hellgren G, Lofqvist C, Hard AL, et al. Serum concentrations of vascular endothelial growth factor in relation to retinopathy of prematurity. Pediatr Res 2016;79(1-1):70-75

24 Kong L, Bhatt AR, Demny AB, et al. Pharmacokinetics of bevacizumab and its effects on serum VEGF and IGF-1 in infants with retinopathy of prematurity. Invest Ophthalmol Vis Sci 2015;56 (02):956-961

25 Peirovifar A, Gharehbaghi MM, Gharabaghi PM, Sadeghi K. Vascular endothelial growth factor and insulin-like growth factor-1 in preterm infants with retinopathy of prematurity. Singapore Med J 2013;54(12):709-712

26 Eichmann A, Simons M. VEGF signaling inside vascular endothelial cells and beyond. Curr Opin Cell Biol 2012;24(02):188-193

27 Turan O, Ergenekon E, Koç E, et al. Impact of phototherapy on vasoactive mediators: NO and VEGF in the newborn. J Perinat Med 2004;32(04):359-364 